\title{
Rapid optical clearing for high-throughput analysis of tumour spheroids
}

\author{
Gency Gunasingh $^{1 *}$, Alexander P Browning ${ }^{2 \dagger}$, Nikolas K Haass ${ }^{1}$ \\ ${ }^{1}$ The University of Queensland Diamantina Institute, The University of Queensland, Brisbane, Australia \\ ${ }^{2}$ School of Mathematical Sciences, Queensland University of Technology, Brisbane, Australia
}

23 November 2021

\begin{abstract}
Tumour spheroids are fast becoming commonplace in basic cancer research and drug development. Obtaining high-quality data relating to the inner structure of spheroids is important for analysis, yet existing techniques often use equipment that is not commonly available, are expensive, laborious, cause significant size distortion, or are limited to relatively small spheroids. We present a high-throughput method of mounting, clearing, and imaging tumour spheroids that causes minimal size distortion. Spheroids are mounted in an agarose gel to prevent movement, cleared using a solution prepared from commonly available materials, and imaged using confocal microscopy. We find that our method yields high quality two- and three-dimensional images that provide information about the inner structure of spheroids.
\end{abstract}

\section{Introduction}

Three-dimensional cell cultures, such as spheroids, provide biologically realistic and reproducible models of aggregate cell growth (Hirschhaeuser et al., 2010). These models are fast becoming commonplace in both basic research and drug development, where differences in spheroid size and structure are examined between treatments to ascertain drug efficacy (Smalley et al., 2007). In these contexts, the ability to collect detailed information from a large number of spheroids is highly advantageous, both from a statistical power perspective, and to allow rapid assessment of cell behaviour across several treatments.

Current techniques for obtaining detailed microscopy images of spheroid structure are either timeconsuming, expensive, or produce poor quality images that do not retain key quantitative features such as spheroid size (Nürnberg et al., 2020; Spoerri et al., 2021). Histological techniques based upon cryosectioning can provide high-quality images, but are often time-consuming or utilise materials that are not readily available (Kabadi et al., 2015). Modern microscopy technologies have relatively recently enabled so-called optical sectioning, where spheroids are placed within a refractive index matched clearing solution and images obtained using confocal microscopy (Nürnberg et al., 2020; Spoerri et al., 2021). While these techniques have the potential to produce

\footnotetext{
g.gunasingh@uq.edu.au

† ap.browning@icloud.com
} 
a high-yield, common problems include spheroid movement, size distortion and high expense of proprietary clearing solutions. Furthermore, many existing protocols apply only to relatively small spheroids, of less than $300 \mu \mathrm{m}$ in diameter, limiting the technology to the early stages of tumour growth.

We present a novel protocol that allows high-throughput, high-yield collection of detailed spheroid images using a low-cost refractive index-matched clearing solution (Susaki et al., 2020). To prevent spheroid movement during imaging and provide structural support to reduce size distortion, we first mount spheroids in agarose-PBS gel in a 24-well \#1.5 glass bottom plate. Since our technique allows for a multiple spheroids to be mounted in each well in a 24-well plate, we can rapidly mount and image large numbers of spheroids across a variety of experimental conditions. To optically clear mounted spheroids and the surrounding gel, we introduce a refractive-indexmatched clearing solution constructed from readily available consumables. After a settling period of 24 hours, our protocol provides high-quality 2D and 3D images of spheroid structure, even for relatively large spheroids (approximately $700 \mu \mathrm{m}$ in diameter), with less than $2 \%$ size distortion.

\section{Protocol}

\section{Materials and consumables}

- Agarose gel (200 $\mu \mathrm{L}$ per 10 spheroids (1 well)).

- Low-melting agarose powder $(2 \% \mathrm{w} / \mathrm{v})$.

- PBS $(98 \% \mathrm{w} / \mathrm{v})$.

- Clearing solution.

- Urea $(22 \% \mathrm{w} / \mathrm{w})$.

- Quadrol® $(9 \% \mathrm{w} / \mathrm{w})$.

○ Sucrose $(44 \% \mathrm{w} / \mathrm{w})$

- Triton X-100 $(0.1 \% \mathrm{w} / \mathrm{w})$

○ MilliQ water $(24.9 \% \mathrm{w} / \mathrm{w})$

- Mounting materials.

○ $750 \mu \mathrm{L}$ clear PCR tubes (1 per well).

○ \#1.5 glass bottom 24-well plate.

\section{Methods}

Methods describe preparation of a sufficient quantity to mount one 24-well plate (approximately 240 spheroids) at $200 \mu \mathrm{L}$ agarose gel per well, $500 \mu \mathrm{L}$ clearing solution per well. Methods are illustrated in Fig. 1. 

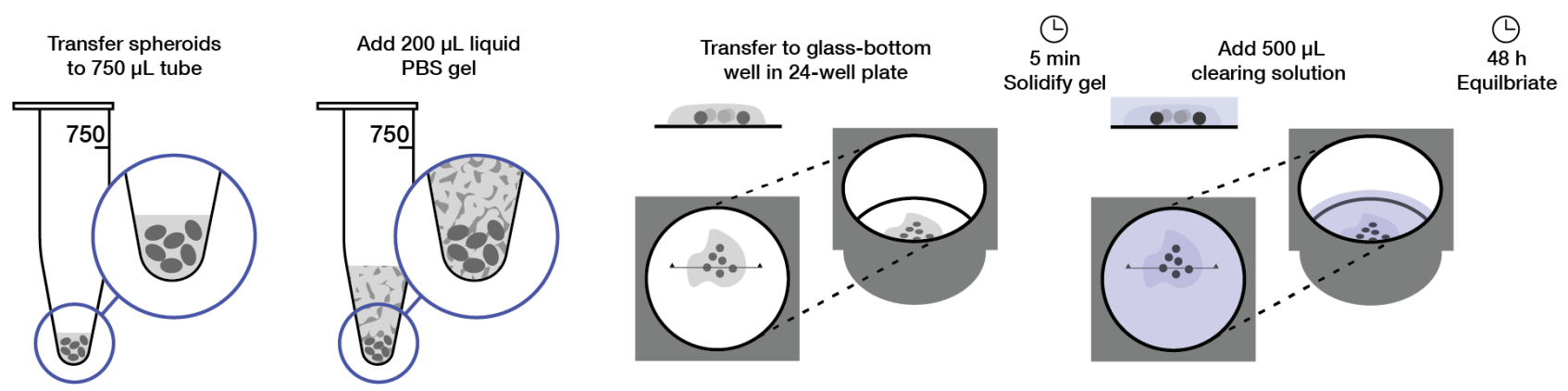

Figure 1. Spheroids are transferred to a $750 \mu \mathrm{L}$ tube, excess fluid is replaced by $200 \mu \mathrm{L}$ PBS-based gel and centrifuged. Spheroids are then transferred to a glass-bottom well in a 24-well plate. After gel is allowed to solidify, $500 \mu \mathrm{L}$ clearing solution is added and spheroids are allowed to equilibrate.

\section{PBS gel preparation}

Dissolve $0.5 \mathrm{~g}$ low-melting agarose in $25 \mathrm{~mL}$ phosphate buffered saline (PBS) by boiling in a microwave for $30 \mathrm{~s}-1 \mathrm{~min}$ with swirling. Ensure agarose is fully dissolved and the solution does not boil over

Gel can be stored at room temperature; check volume before use to account for evaporation.

Bring to liquid state (microwave; $20 \mathrm{~s}-1 \mathrm{~min}$, with swirling) before using.

\section{Clearing solution preparation}

Weigh Quadrol@ and adjust the weight of other components to obtain desired concentrations. Weigh desired quantities of all components of the clearing reagent, including water, and heat up the solution in $56^{\circ} \mathrm{C}$ water bath with constant mixing. Once mixed, degas the solution or allow the bubbles to rise to surface prior to use. The solution is stable at room temperature up to 2 months.

\section{Mounting}

1. Transfer fixed and stained spheroids to $750 \mu \mathrm{L}$ PCR tubes at one tube per condition (approximately 10 spheroids). Let spheroids gravity settle to bottom of the tube.

2. Replace solution with $200 \mu \mathrm{L}$ liquid agarose gel and centrifuge for $30 \mathrm{~s}$. Unless mounting immediately, place in heating block at $50{ }^{\circ} \mathrm{C}$ to avoid gel hardening.

3. Aspirate spheroids in $\sim 50 \mu \mathrm{L}$ liquid agarose gel. Dispense in well of a 24 -well glass bottom plate. Before gel hardens, separate spheroids using pipette tip in surrounding gel, and ensure spheroids are covered with gel. Optionally, place plate on ice to rapidly set gel.

4. Add $500 \mu \mathrm{L}$ clearing solution per well, ensuring gel is submerged. Leave for $>24 \mathrm{~h}$ before imaging.

\section{Imaging}

1. Choose an objective that has a working distance long enough to encompass the entire spheroid including the mounting height of the spheroid in the vessel. 
2. For equatorial section, adjust focus till the largest surface area is reached in the $X Y$ plane and image with required laser power, detector voltage, gain and offset settings.

3. For 3D images, set start and end of the spheroids, choose appropriate signal intensity at various Z-depth using Z intensity correction settings (Bright Z in FV3000) before imaging.

4. For best image resolution, use Nyquist sampling rate for $X, Y$ and $Z$.

All images shown in this paper were imaged using Olympus FLUOVIEW FV3000 confocal microscope.

(a)

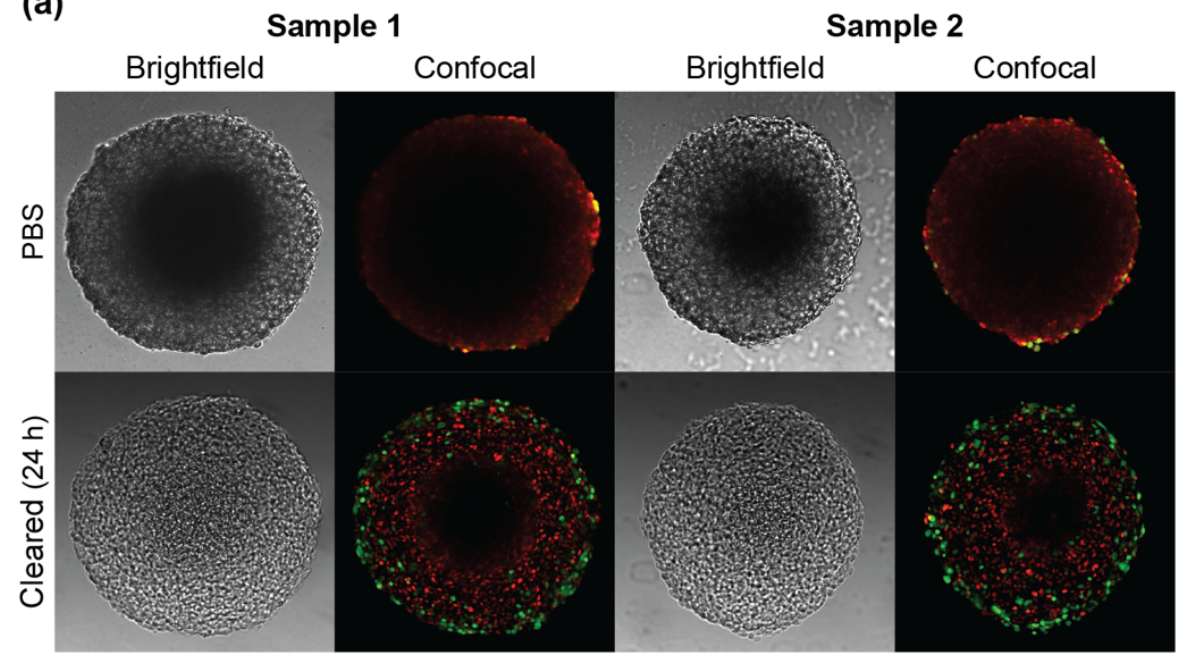

(c)

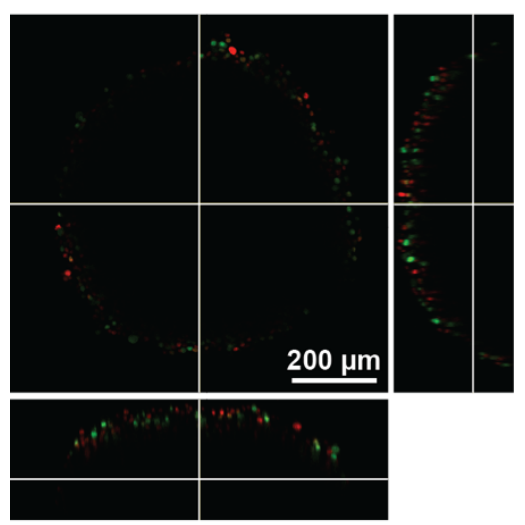

Cleared (24 h)

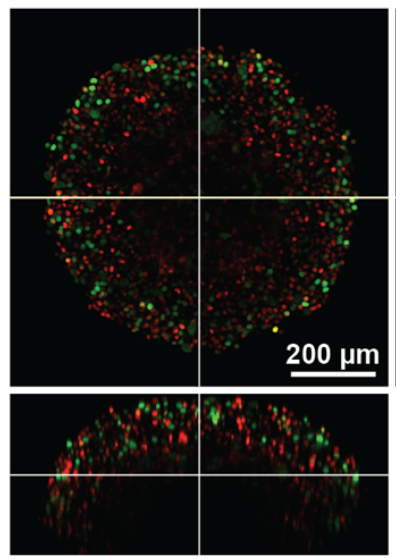

(b)

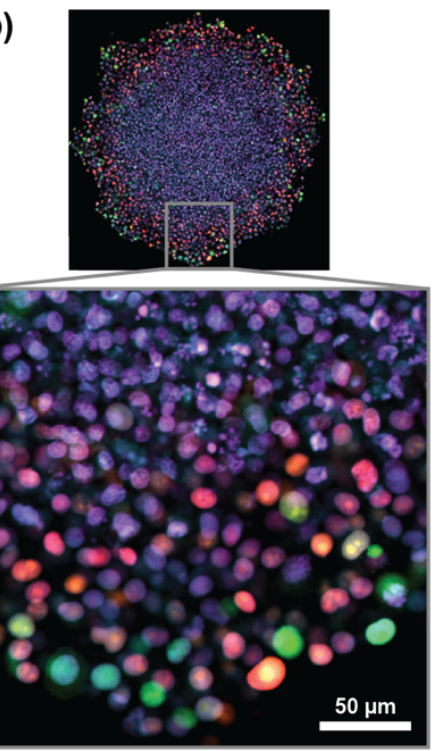

Cleared $(24 \mathrm{~h})$ with bright-z
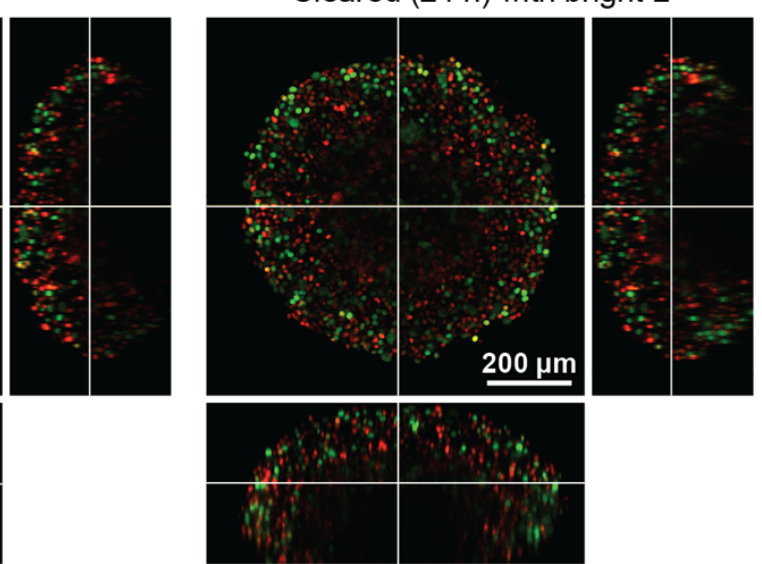

Figure 2. Confocal cross-sectional images obtained of spheroids grown from human melanoma cells transduced with fluorescent cell cycle indicators (FUCCI) (Sakaue-Sawano et al., 2008; Haass et al., 2014). Spheroids are grown using methods described in Spoerri et al. 2017 Colouring indicates cell nuclei positive for mKO2 (red), which indicates cells in gap 1; and cell nuclei positive for mAG (green), which indicates cells in gap 2. (a) Spheroids grown from 5000 FUCCI-WM983b cells (Haass et al., 2014), harvested at day 10 and imaged in PBS gel and $24 \mathrm{~h}$ after clearing solution is added. Comparing brightfield and confocal images before and after clearing solution shows minimal size distortion, and a large gain in clarity. Images are obtained using 10x objective. (b) Spheroid grown from FUCCI-WM164 cells (Haass et al., 2014) permeabilised using Triton and stained with DRAQ7, which stains all cell nuclei. Image is obtained using a 20x objective, demonstrating the clearing solution allows high-resolution image of cell-level details. (c) Three-dimensional images obtained of FUCCI-WM164 spheroids in PBS and $24 \mathrm{~h}$ after clearing solution is added. We apply bright-z to adjust exposure settings for cross-sections obtained deeper inside the spheroid. Cleared images still show light loss due to scattering from the necrotic core. 


\section{Results and Discussion}

To demonstrate the ability of our clearing method to provide high-quality two- and threedimensional images, we mount, clear and image relatively large spheroids grown from human melanoma cell lines according to methods given in Spoerri et al., 2017 (Fig. 2). All microscopy files are provided on GitHub (see Data Availability). Compared with PBS-mounted spheroids, the clearing solution provides high-clarity images with minimal size distortion (Fig. 2a). In Fig. 2b, we demonstrate that the protocol allows for high-resolution imaging of cell-level details without histological sectioning; this cross-sectional image was obtained from a 20x air objective (Olympus UPlanSApo) at a resolution of $4096 \times 4096$ px without stitching. In Fig. 2c, we demonstrate threedimensional confocal images that provide cell-level detail at a depth of at least $200 \mu \mathrm{m}$. Note that Bright-Z in FV3000, which provides a depth-based intensity correction in Z, allows the imaging of the whole spheroid. We note that light-scattering due to the necrotic core limits our ability to image the far side of the spheroid.

To determine whether the clearing solution causes size distortion, we image 12 spheroids in PBS gel, and $6 \mathrm{~h}, 12 \mathrm{~h}, 24 \mathrm{~h}, 72 \mathrm{~h}$ and $168 \mathrm{~h}$ following the introduction of the clearing solution. We summarise images by determining the diameter of the spheroid, defined based on a sphere with the same cross-sectional area as the spheroid (Fig. 3a). While the spheroids are observed to slightly increase in size over the first $6 \mathrm{~h}$, indicated by a diameter fold-change of between $-2 \%$ and $6 \%$ (Fig. 3b), after 24 to $72 \mathrm{~h}$ the spheroids return to a size approximately equal to the corresponding size in PBS gel only (Fig. 3c). Combined with automated image processing (Browning et al., 2021a) and minimal size distortion, our high-throughput protocol lends to quantitative analysis of spheroid inner structure (Browning et al., 2021b).

(a)

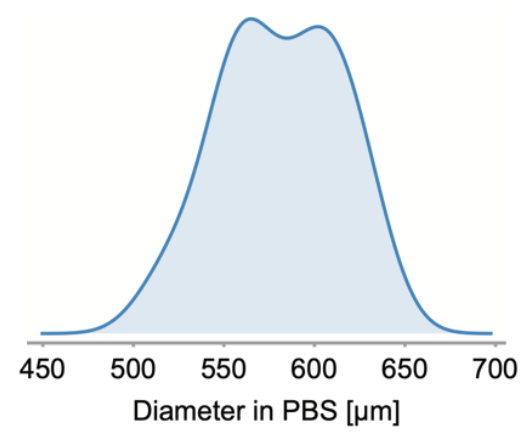

(b)

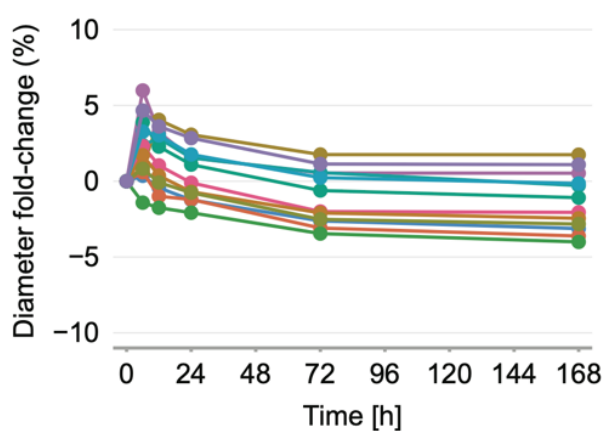

(c)

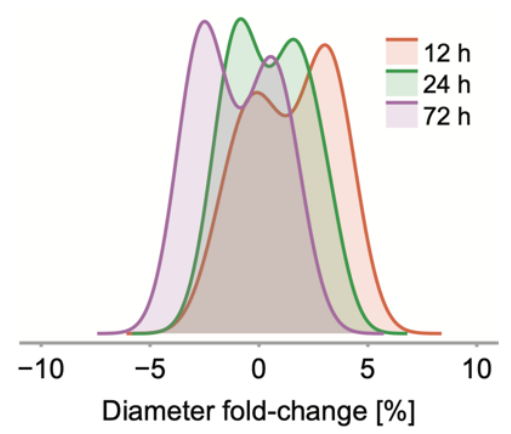

Figure 3. Clearing solution has very little impact on spheroid size. (a) Distribution of initial spheroid size (equivalent diameter) in PBS gel ( $n=12$ spheroids). (b) Diameter fold-change over time since addition of clearing solution. At 0 hours, spheroids are in PBS gel only. (c) Distribution of diameter fold-changes at $12 \mathrm{~h}, 24 \mathrm{~h}$ and $72 \mathrm{~h}$. 


\section{Conclusion}

We present a high-throughput protocol for obtaining high-quality two- and three-dimensional images of tumour spheroids. Our method uses readily available consumables to mount and clear spheroids that maintains optical clarity while preventing size distortion. Given that our technique allows multiple spheroids to be mounted simultaneously in a multi-well plate, our protocol is well suited to quantitative analysis pipelines that require spheroid structure information from large numbers of spheroids.

\section{Data availability}

Microscopy images and code used to perform spheroid size analysis are available on GitHub at https://github.com/ap-browning/SpheroidMounting.

\section{Acknowledgements}

This research was carried out at the Translational Research Institute (TRI), Woolloongabba, QLD. TRI is supported by a grant from the Australian Government. We thank the staff in the microscopy core facility at TRI for their outstanding technical support. We thank Prof. Atsushi Miyawaki, RIKEN, Wako-city, Japan, for providing the FUCCI constructs, Prof. Meenhard Herlyn and Ms. Patricia Brafford, The Wistar Institute, Philadelphia, PA, for providing the cell lines.

\section{Funding}

This work was supported by project grants to N.K.H.: Australian Research Council (DP200100177) and Meehan Project Grant (021174 2017002565). 


\section{Bibliography}

Browning AP \& Murphy RJ. Image processing algorithm to identify structure of tumour spheroids with cell cycle labelling. Zenodo (2021a) doi:10.5281/zenodo.5121093.

Browning AP, Sharp JA, Murphy RJ, Gunasingh G, Lawson B, Burrage K, Haass NK \& Simpson MJ. Quantitative analysis of tumour spheroid structure. bioRxiv (2021b) doi:10.1101/2021.08.05.455334.

Haass NK, Beaumont KA, Hill DS, Anfosso A, Mrass P, Munoz MA, Kinjyo I \& Weninger W. Real-time cell cycle imaging during melanoma growth, invasion, and drug response. Pigment Cell Melanoma Research 27, 764-776 (2014).

Hirschhaeuser, F., Menne, H., Dittfeld, C., West, J. \& Mueller-Klieser W. Multicellular tumor spheroids: an underestimated tool is catching up again. Journal of Biotechnology 148, 3-15 (2010).

Ivanov DP \& Grabowska AM. Spheroid arrays for high-throughput single-cell analysis of spatial patterns and biomarker expression in 3D. Scientific Reports 7, 41160 (2017).

Kabadi PK, Vantangoli MM, Rodd AL, Leary E \& Madnick SJ. Into the depths: techniques for in vitro threedimensional microtissue visualization. Biotechniques 59, 279-286 (2015).

Nürnberg E, Vitacolonna M, Klicks J, von Molitor E \& Cesetti T. Routine optical clearing of 3D-cell cultures: simplicity forward. Frontiers in Molecular Biosciences 7, 20 (2020).

Sakaue-Sawano A, Kurokawa H, Morimura T, Hanyu A \& Hama H. Visualizing spatiotemporal dynamics of multicellular cell-cycle progression. Cell 132, 487-498 (2008).

Smalley K, Lioni M, Noma K, Haass NK \& Herlyn, M. In vitro three-dimensional tumor microenvironment models for anticancer drug discovery. Expert Opinion on Drug Discovery 3, 1-10 (2007).

Spoerri L, Beaumont KA, Anfosso A \& Haass NK. Real-time cell cycle imaging in a 3D cell culture model of melanoma. Methods in Molecular Biology 1612, 401-416 (2017).

Spoerri L, Gunasingh G \& Haass NK. Fluorescence-based quantitative and spatial analysis of tumour spheroids: a proposed tool to predict patient-specific therapy response. Frontiers Digital Health 3, 668390 (2021).

Susaki EA, Shimizu C, Kuno A, Tainaka K \& Li X. Versatile whole-organ/body staining and imaging based on electrolyte-gel properties of biological tissues. Nature Communications 11, 1982 (2020). 